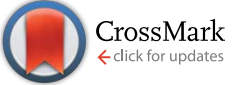

Cite this: RSC Adv., 2016, 6, 15877

Received 7th January 2016

Accepted 26th January 2016

DOI: 10.1039/c6ra00493h

www.rsc.org/advances

\section{A facile method of synthesizing size-controlled hollow cyanoacrylate nanoparticles for transparent superhydrophobic/oleophobic surfaces $\uparrow$}

\author{
Takeshi Matsubayashi, ${ }^{a}$ Mizuki Tenjimbayashi, ${ }^{a}$ Kengo Manabe, ${ }^{a}$ Kyu-Hong Kyung, ${ }^{a}$ \\ Bin Ding ${ }^{\mathrm{b}}$ and Seimei Shiratori*a
}

Hollow nanoparticles have broad technological implications in a wide range of applications. Particularly, they have attracted great attention as functional coatings in applications such as optical devices, which have an optical transparency derived from a low refractive index. However, creating a facile and versatile method that can accurately control the hollow nanoparticle size has proven extremely challenging. Herein, we report a simple, instantly complete, one-pot method, designated the supersaturated gascored instant polymerization (SGCIP) method, to synthesize size-controlled hollow cyanoacrylate nanoparticles (HCNPs). The SGCIP method uses supersaturated gas created by mixing two solvents (water and acetone) and the instant polymerization of cyanoacrylate, whereby it demonstrates facile control of the particle diameters ranging from 13 to $1830 \mathrm{~nm}$ reproducibly by simply changing the solvent ratio. Moreover, a unique phase transition (from network to particle formation) is observed during the adjustment of the solvent ratio. As a one-concept application, transparent superhydrophobic/ oleophobic coatings are achieved by self-assembly of the HCNPs and silanization. The successful synthesis of such fascinating materials may also provide new insights into the design and development of functional hollow nanoparticles for various applications.

\section{Introduction}

The synthesis of hollow nanoparticles has attracted much attention because of the unique morphology of these nanoparticles, such as the inner void structure, the high surface-tovolume ratio, and high porosity., ${ }^{1,2}$ These unique characteristics allow hollow nanoparticles to have many potential applications in areas such as drug delivery, ${ }^{3,4}$ catalysis, ${ }^{5}$ and optical materials. ${ }^{6,7}$ To synthesize hollow nanoparticles, typically the core materials inside the particle shells are removed through combustion or dissolution. ${ }^{8,9}$ Caruso et al. have proposed a fabrication method for hollow spheres with diameters between 720 and $1000 \mathrm{~nm}$ via layer-by-layer adsorption and calcination..$^{10}$ In addition, Du et al. demonstrated the utility of hollow silica and fabricated antireflection coatings using the

${ }^{a}$ School of Integrated Design Engineering, Center for Science and Technology for Designing Functions, Graduate School of Science and Technology, Keio University, 3-14-1 Hiyoshi, Kohoku-ku, Yokohama, Kanagawa 223-8522, Japan. E-mail: shiratori@appi.keio.ac.jp; Fax: +81-45-566-1602; Tel: +81-45-566-1602

${ }^{b}$ State Key Laboratory for Modification of Chemical Fibers and Polymer Materials, College of Materials Science and Engineering, Donghua University, Shanghai 201620, China.E-mail: binding@dhu.edu.cn

$\dagger$ Electronic supplementary information (ESI) available: SEM images of HCNPs not shown in the main text, particle diameter distribution, particle diameter measured by SEM images, XPS results, results of verification test (SEM images) and Movie S1 and S2. See DOI: 10.1039/c6ra00493h dissolution process, and they have also indicated that the introduction of hollow particles was a promising approach for transparent coatings. ${ }^{11}$ However, this core-removal step often damages the nanoparticles, makes the entire procedure more complicated, and makes it difficult to control the particle size.

Here, we demonstrate a novel and facile method to fabricate size-controlled hollow polymer nanoparticles composed of an air core and a polymer shell. The air phase of the nanoparticle core is derived from supersaturated gas that occurs by mixing two solvents with different gas solubilities. ${ }^{12}$ Ethyl cyanoacrylate (ECA) is selected as the shell material for the hollow nanoparticles because of its high reactivity and strong adhesive properties. ${ }^{13,14}$ This method for fabricating hollow cyanoacrylate nanoparticles (HCNPs) is designated as the supersaturated gascored instant polymerization (SGCIP) method, which uses both the supersaturated gas produced by mixing two solvents and the instant reaction of ECA. The gas-cored polymer structures created via the SGCIP process can predominantly manifest either as a network structure or an assembled particle structure by changing the ratio of the solvents. The boundary of the inversion between network and particle structure is also investigated. In the particle structure, the diameters of the HCNPs can be readily controlled by simply changing the ratio of the solvents. This novel method is completed within a few minutes in a wet process and without using any special devices. 
Synthesized HCNPs have been applied to transparent antifouling surfaces, thereby mimicking the surface structure of superhydrophobic lotus leaves, which are of great interest in terms of their attributes of self-cleaning, ${ }^{15,16}$ anti-icing, ${ }^{17,18}$ and oil/water separation, ${ }^{\mathbf{1 9 - 2 1}}$ among others. It is significant that the micro-scale roughness of superhydrophobic surfaces causes Mie light scattering, which leads to a decrease of optical transmittance in the visible range. ${ }^{22,23}$ This scattering becomes more marked for surfaces designed to repel oil because oleophobicity requires higher roughness. ${ }^{24,25}$ Therefore, inspired by the hawkmoth cephonodes hylas, ${ }^{26}$ HCNPs have been used to produce transparent superhydrophobic/oleophobic surfaces that solve the problem of the conflicting states of transparency and superhydrophobicity/oleophobicity. The wings of the hawkmoth are optically transparent as a protection mechanism against enemies, and the transparency is accomplished by the hollow nanostructure of their wings that produces a low refractive index and suppresses surface light scattering. ${ }^{27}$ Recently, $\mathrm{Xu}$ et al. prepared hollow silica nanoparticles to fabricate transparent superhydrophobic surfaces, ${ }^{28}$ and Gao et al. have similarly reported transparent superhydrophobicity with hollow silica nanoparticles; ${ }^{29}$ yet there is room for development in the field of transparent anti-fouling coatings in terms of particle size control and the time cost of the process. In this article, size-controlled HCNPs are synthesized via a facile SGCIP method and coated onto substrates, followed by a silanization treatment. The coated surfaces exhibit varying wettability and high transparency as the particle diameters are varied. Hollow nanoparticles synthesised in our process demonstrate an application for optical coatings that may contribute to the coexistence of the typically conflicting properties of transparency and superhydrophobicity or oleophobicity, and show the possibility of the development of not only transparent anti-fouling coatings but also drug delivery application or other functional coatings.

\section{Experimental section}

\section{Materials}

Ethyl cyanoacrylate (purity: 99.9\%, Kobunshi Shouji Co. Ltd., Osaka, Japan) and acetone (Kanoto Chemical Co., Inc., Tokyo, Japan) were used for the preparation of the HCNPs. Deionized water with a resistivity higher than 18.2 $\mathrm{M} \Omega$ was used in all experiments and was obtained from a three-stage Millipore Mill-Q Plus 185 purification system (Academic). The 2-propanol (IPA), hydrochloric acid (Kanoto Chemical Co., Inc., Tokyo, Japan), tetraethyl orthosilicate (TEOS) (Wako Pure Chemical Industries Ltd., Osaka, Japan), (heptadecafluoro-1,1,2,2tetrahydrodecyl) triethoxysilane as fluoroalkyl silane (FAS) (Gelest Inc., Morrisville, PA, USA), glass substrates $(76 \times 26$ $\mathrm{mm}^{2}$, thickness $=1.0 \mathrm{~mm}$, refractive index $=1.52$; Matsunami Glass Ind., Ltd., Kishiwada, Japan), and sprayer (spray nozzle = $0.6 \mathrm{~mm}$, spray pressure $=0.7 \mathrm{MPa}$; Airtex Co., Ltd., Osaka, Japan) were used for film fabrication and low surface energy treatment. Activated carbon (Wako Pure Chemical Industries Ltd., Osaka, Japan) and oleic acid (Tokyo Chemical Industry
Co., Ltd., Tokyo, Japan) were mixed and used as an artificial fingerprint liquid.

\section{Synthesis of the HCNP solution by the SGCIP method}

The ECA ( $0.1 \mathrm{~g})$ was dissolved into a certain amount of acetone, whereupon water was dropped with a micropipette into the ECA solution under ultra-sonication with oscillatory frequency of 42 $\mathrm{kHz}$ and output power of $80 \mathrm{~W}$ at the static temperature of 25 ${ }^{\circ} \mathrm{C}$. The water adding rate was $5 \mu \mathrm{L} \mathrm{s}{ }^{-1}$. To easily discuss the synthesis mechanisms, the water ratio (WR) is defined as the following equation;

$$
\mathrm{WR}=\frac{\text { weight of added water }}{\text { total weight of water and acetone }}
$$

The amount of acetone and water was changed as a varying water ratio (WR) with a total amount of acetone and water of $10.0 \mathrm{~g}$. The WR was varied from 0 to $30.0 \mathrm{wt} \%$. When WR is 10.0 $\mathrm{wt} \%$, for example, $1 \mathrm{~g}$ of water is dropped, thus it takes $200 \mathrm{~s}$ to complete water addition. Water adding time varied for WR.

\section{HCNP coating on a glass substrate}

The HCNP solution $(2.0 \mathrm{~mL})$ was sprayed at a spray distance of $40 \mathrm{~cm}$ onto glass substrates cleaned with acetone. The WR of the HCNP solution was adjusted to be 5.0, 7.0, 10.0, 15.0, 20.0, 25.0 , and $30.0 \mathrm{wt} \%$.

\section{Silanization of the HCNP-coated surfaces with FAS}

The post-silanization process of the HCNP-coated surfaces was conducted by simply immersing samples into a FAS solution. After stirring the mixing solution of IPA $(38.4 \mathrm{~mL})$, TEOS $(0.50$ $\mathrm{g})$, FAS $(0.50 \mathrm{~g}), \mathrm{HCl}(50 \mu \mathrm{L})$, and $\mathrm{H}_{2} \mathrm{O}(250 \mu \mathrm{L})$ for $1 \mathrm{~h}$, the HCNPcoated surfaces were immersed in the mixture for $24 \mathrm{~h}$.

\section{Characterization}

The diameters of the synthesized particles were measured by dynamic light scattering (DLS; ELS-8000, Otsuka Electronics Co., Ltd., Osaka, Japan). Field emission scanning electron microscope (FE-SEM) images were taken using a FE-SEM (S4700, Hitachi, Tokyo, Japan) with an accelerating voltage of 10 $\mathrm{kV}$ to characterize the surface morphologies of the films. For transmission electron microscopic (TEM) observation, an HCNP solution of WR $=10.0 \mathrm{wt} \%$ was cast onto carbon-coated copper grids (COL-C10, Okenshoji Co., Ltd., Tokyo, Japan) and dried overnight, whereupon the TEM (Tecnai G2, FEI, Hillsboro, OR, USA) with an acceleration voltage of $200 \mathrm{kV}$ was used to obtain images of the HCNPs. Transmittance measurements in the spectral range of 300-1000 nm were carried out using a spectrophotometer (UVmini-1240, Shimadzu, Kyoto, Japan), while contact and sliding angles were measured using a contactangle meter (CA-DT, Kyowa Interface Science Co., Ltd., Niza, Japan). Optical values were measured by a haze meter (NDH5000, Nippon Denshoku Industries, Tokyo, Japan) with a white light-emitting diode $(5.0 \mathrm{~V}, 3.0 \mathrm{~W})$ as an optical source. The concept of each optical value is defined as follow. Parallel 
transmittance (P.T.) means the degree that light passes through a sample within a small range of angle $\left(<15^{\circ}\right)$ and diffusion (DIF) is a scattering of light when it passes through the sample. Total transmittance (T.T.) is the sum of P.T. and DIF. HAZE is calculated as $(\mathrm{DIF} / \mathrm{T} . \mathrm{T}) \times .100 .^{30} \mathrm{X}$-ray photoelectron spectroscopy (JPS-9010TR, JEOL, Tokyo, Japan) with an MGK $\alpha$ laser was used to investigate the chemical composition of the surface.

\section{Fingerprint adhesion test}

Activated carbon (10 mg) was dispersed into oleic acid (2.0 g) under ultra-sonication to create the artificial fingerprint material, $30 \mu \mathrm{L}$ of which was cast on a rubber stamp $\left(2 \times 2 \mathrm{~cm}^{2}\right)$ that was pressed onto the bare glass and the coated glass with a pressure of $12 \mathrm{kPa}$. The color coordinates were measured to calculate color differences using a color difference meter (Color Reader CR-13, Minolta Co., Ltd., Tokyo, Japan), which were calculated by comparing the results obtained before and after applying an artificial fingerprint to the glass (bare and coated).

\section{Results and discussion}

\section{Synthesis mechanism of HCNPs via the SGCIP method}

There are some studies reporting fabrication methods of cyanoacrylate nanoparticles. ${ }^{\mathbf{3 1 , 3 2}}$ In this study, HCNPs are synthesized by a novel one-step process combining two reactions via the SGCIP method (schematic shown in Fig. 1a). Since acetone has low polarity than water, acetone has higher solubility to nonpolar gas like oxygen or nitrogen. ${ }^{33}$ When a polar and a nonpolar solvent, such as water and acetone, are blended to form a mixture, gas solubility of the mixture is smaller than the sum of gas solubility of water and acetone, thus supersaturated gas comes out of the solution in the form of nanobubbles until the concentration is at the saturation level (reaction I).$^{12}$ In addition, ethyl cyanoacrylate (ECA), commonly used as "super glue" and surgery adhesive, forms a strong bond to various materials instantly reacting under humid atmosphere. ${ }^{34}$ ECA monomers are soluble and not polymerized in acetone but are instantly polymerized by nucleophilic-initiated mechanisms in the presence of water (scheme shown in Fig. S1†) (reaction II). ${ }^{35,36}$ As a result, HCNPs are obtained through reactions I and II occurring simultaneously. In reaction I, adding water to acetone results in the nucleation of supersaturated gas regardless of whether or not ECA is dissolved in the acetone. ${ }^{37}$ In reaction II, ECA polymerization occurs at the interface of the gas, water, and acetone and surrounds the gas inside. The ECA monomer has a hydrophilic cyano $(-\mathrm{CN})$ group that is oriented to the acetone and a relatively hydrophobic-COOEt group that is oriented to the gas. In our hypothesis, a water/acetone mixture exists at the gas surface, while the outside is filled solely with acetone when the supersaturated gas nucleates. ${ }^{38}$ Hence, a gas surface interacts more easily with the -COOEt group and orients like a surfactant in the water/oil mixture. ${ }^{39}$ This is how HCNPs are formed with the multi-interface interaction.

The synthesized HCNPs are observed by digital microscopy (Fig. 1b) and transmission electron microscopy (TEM, Fig. 1c), which indicates that the particle is composed of two parts; an air core and a surrounding shell polymer layer, where the air core is derived from the gas created by mixing acetone and water. On the other hand, particles with not hollow structure are also observed (Fig. S2 $\dagger$ ). This result is due to the local reaction of bubble generation, yet the ratio of hollow particles is not confirmed and there still remains room for further investigation of HCNPs at this time.

\section{Structure control of HCNPs}

We vary the amount of acetone and water, and thereby vary the WR, to observe the morphology and particle size of the HCNPs, where the diameter of the HCNPs is analyzed by dynamic light scattering (DLS). As the WR is increased, the dispersed HCNP solution changes from colorless to opaque (Fig. 2a inset) resulting from the increased light scattering (Fig. 2a) as
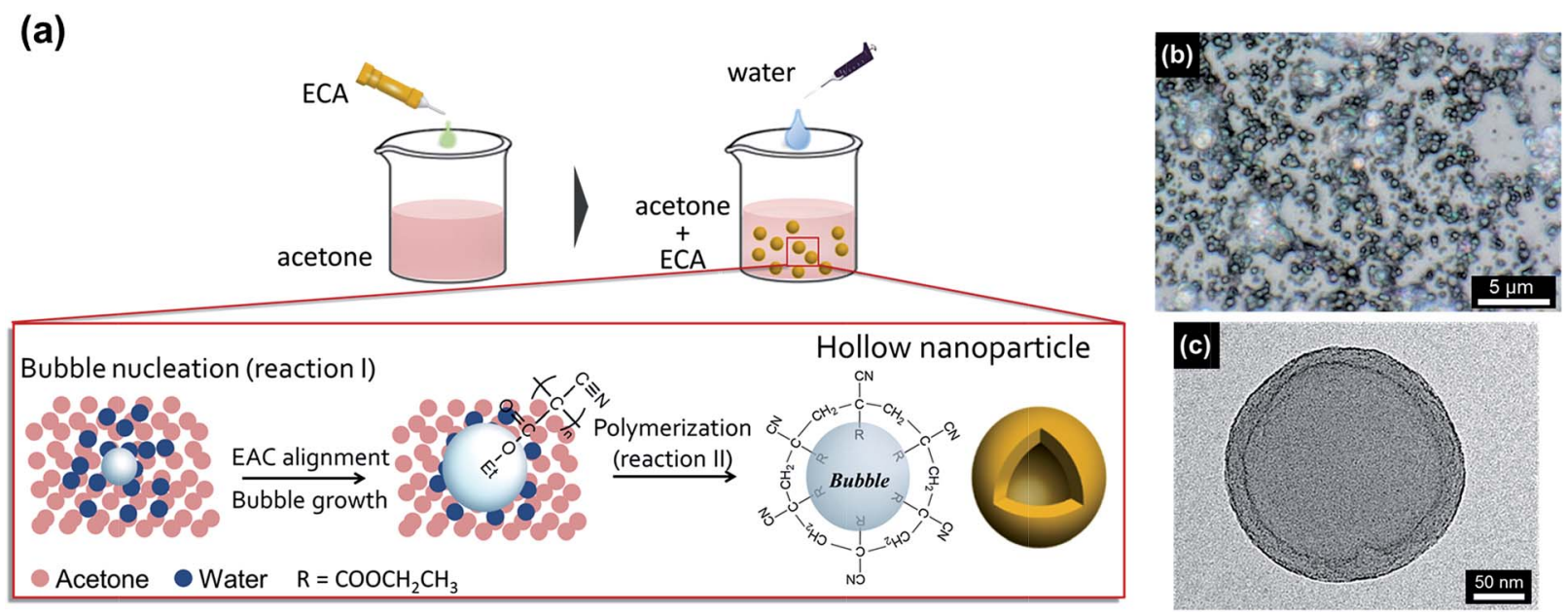

Fig. 1 (a) Schematic image of the mechanism of HCNPs generation from microscopic view of point. (b) Digital microscopic of HCNP coated glass substrate by spraying method and (c) TEM images of HCNPs. WR are (b) 30.0 and $($ (c) 10.0 wt\%. To better observe HCNPs by digital microscope, large particles (WR $=30.0 \mathrm{wt} \%$ ) were prepared. 
(a)

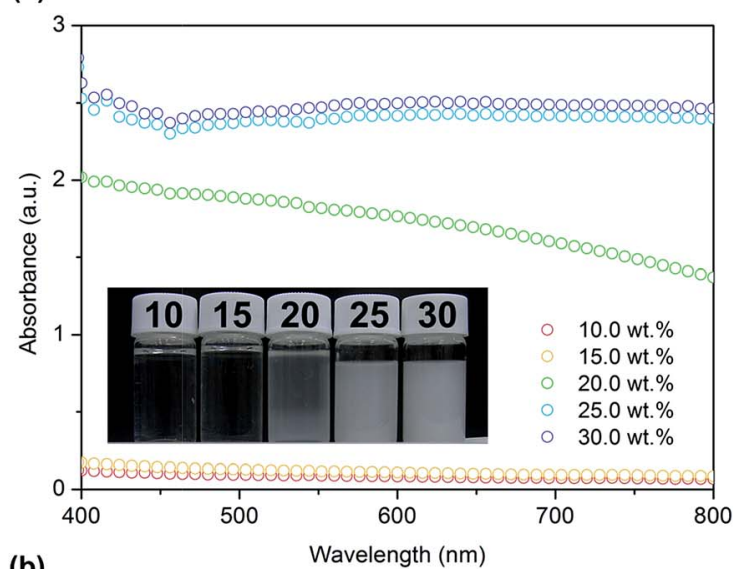

(b)

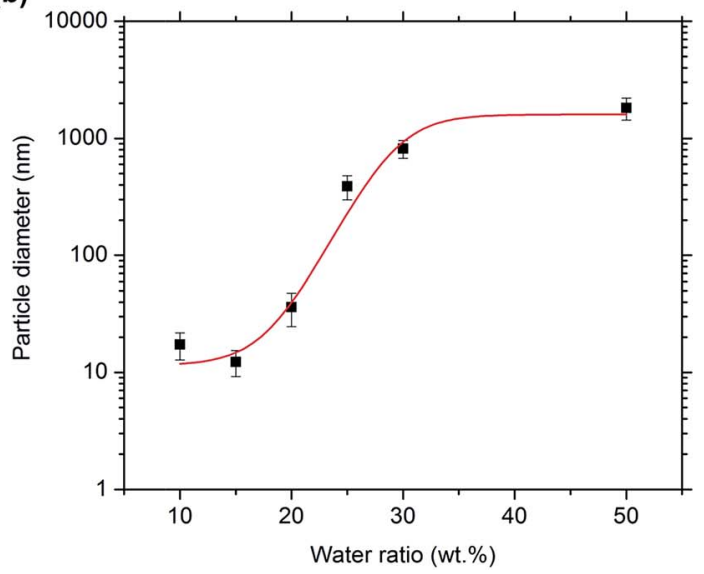

Fig. 2 (a) UV-vis absorption spectra of HCNP solution with different WR. From lower to upper curve, WR is 10.0, 15.0, 20.0, 25.0 and 30.0 wt\%. (b) Particle diameter of HCNPs at different WR. Red curve in the figure is drawn by Boltzmann fitting. Dispersion of particle diameters conform a Gaussian distribution and error bars for each plot are standard deviation of the distribution (see Fig. S3 $\uparrow$ ).

a function of the increased size of HCNPs, as confirmed by the particle diameter measurements in Fig. $2 \mathrm{~b}$ (particle size distributions are given in Fig. S3†). Fig. 3a-d exhibits the scanning electron microscope (SEM) images of HCNPs resulting from each WR $(0,7.0,10.0,30.0 \mathrm{wt} \%)$. To focus on the particlestructured HCNPs, the dispersed solution was cast onto a glass substrate and then observed by SEM (Fig. S4†). The particle diameters in the SEM images, wherein the HCNPs are dry and are under vacuum and exposed to an electron beam, differ slightly from those measured by DLS (Fig. S5 $\dagger$ ), and it is considered that HCNPs with very small diameters $(<40 \mathrm{~nm})$ are broken under the vacuum conditions in SEM because the shell walls are too thin to endure the inner pressure of hollow particles.

It is suggested by the results of both DLS (Fig. 2b) and the SEM images (Fig. S4 $\dagger$ ) that the structure of the HCNPs can be controlled by varying the WR, where it is observed that the size of the HCNPs increases exponentially as the WR is changed from 15.0 to $30.0 \mathrm{wt} \%$. A build-up of the polymer shell is believed to cause this growth in the particle diameters because the ECA polymerizes exponentially. Because the water is constantly added, the as-synthesized HCNPs in the solution play the role of cores in the subsequent ECA polymerization.

To explore this further, the curve of the particle size as a function of the WR in Fig. 3 is noteworthy, where it is seen to approach an equilibrium curve (Fig. $2 \mathrm{~b}$ ) because of the synthesis mechanism of the HCNPs. As demonstrated in the ESI Movie S1, $\uparrow$ the synthesized HCNPs disappear in the acetonerich solution and appear again in the relatively water-rich solution. Therefore, it seems that the ECA in the water/ acetone mixture solvent is reversibly dissolved and polymerized to some extent at any WR, which means SGCIP is an equilibrium-like reaction.

Moreover, HCNPs cannot be observed as a form of particles when the WR is less than $10.0 \mathrm{wt} \%$ (Fig. 3a and b), but instead a connected and continuous polymer structure is observed when $\mathrm{WR}=0$ and $7.0 \mathrm{wt} \%$. The reason of this network structure formation is considered to be driven by the high solubility of acetone to ECA. We guess these network structures are formed by partial coalescence of HCNPs. When the ratio of acetone is high, outermost surface of HCNPs are dissolved in acetone to adhere each other driven by self-assembly of particles. Then the dissolved ECA is again solidified and water-existed area is extracted when cast on glass slides and left on the atmosphere. For WR $=0 \mathrm{wt} \%$, ECA is polymerized by water in the atmosphere and pores are formed by the extraction of this water. On the other hand, at WR is over $10.0 \mathrm{wt} \%$, HCNPs are not so much dissolved as retaining the particle structure because of less existence of acetone as a solvent of ECA.

Fig. S6a-c $\dagger$ shows the further investigation of the changing point from a network structure to particles where the changing point occurs at a WR value of $10.0 \mathrm{wt} \%$. Moreover, we vary the amount of ECA in addition to varying the WR (water-to-acetone ratio) (Fig. S7a-f, S8a-c $\dagger$ ), where the ECA/water/acetone ternary phase diagram examined in this study and the phase inversion border is mapped in Fig. $3 \mathrm{f}$.

Furthermore, SGCIP method has potential to be applied for other solvent mixture system. The system should have monomer, solvent and initiator, which instantly polymerizes the monomer. It is also required that the polarity of solvent and initiator have some difference, which leads to the difference of gas solubility and thus supersaturated gas occurrence. When the three materials meet above requirement, SGCIP method can be worked and hollow particles are possibly obtained.

\section{Surface wettability and optical transparency}

Transparent superhydrophobic/oleophobic surfaces are fabricated by spraying HCNPs onto glass and following silanization, where the morphology of the HCNP-coated surfaces is shown in Fig. S9a-g. $\dagger$ In addition, a surface elemental analysis of the HCNP (WR $=20.0 \mathrm{wt} \%$ )-coated surfaces before and after silanization is conducted (Fig. 4a and b, S10a and b†). The peak of nitrogen ( $\mathrm{N} \mathrm{1s,} 398 \mathrm{eV}$ in theory) as derived from the cyano $(-\mathrm{CN})$ group is seen to disappear while the peak of fluorine (F 1s, $685 \mathrm{eV}$ in theory) as derived from fluoroalkylsilane (FAS) is seen to intensify after silanization. These results confirm that the films are silanized and are covered by FAS. 


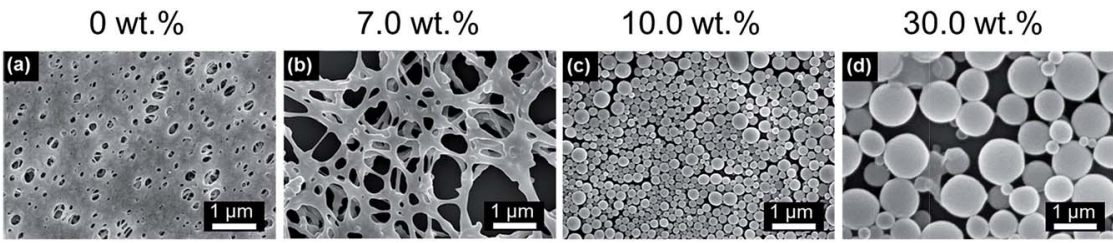

(e)

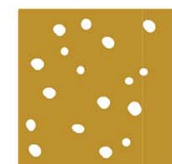

ECA

Network structure
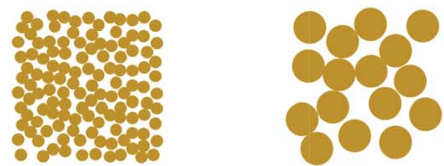

Particle structure (f)
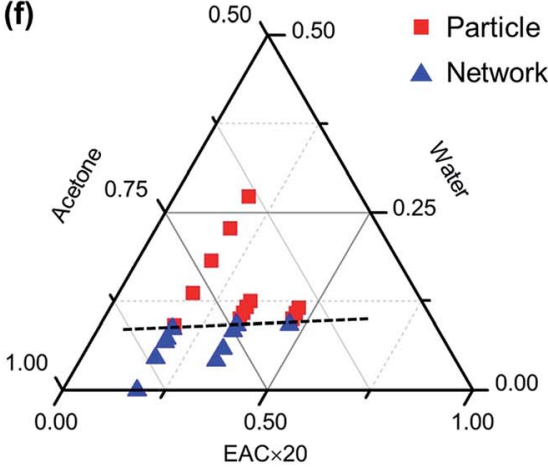

WR

Fig. 3 Scanning electron microscope images showing the structure and size changes of hollow cyanoacrylate nanoparticles fabricated using a water ratio of (a) 0 , (b) 7.0, (c) 10.0, and (d) 30.0 wt\%. (e) Corresponding schematics showing the transition of the phase separation types as a function of changing water ratio. (f) Phase diagram of the ethyl cyanoacrylate/acetone/water ternary system as a function of the weight ratio, marking the boundary between network and particle structure (dashed line).

(a)

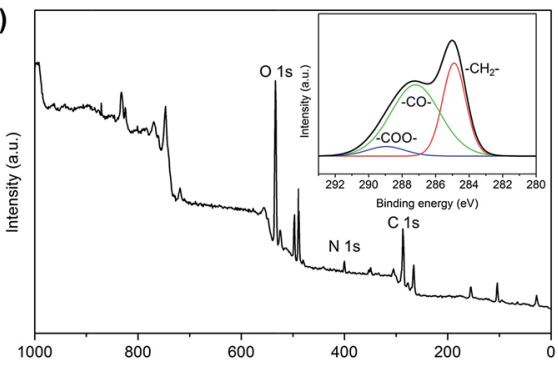

(b)

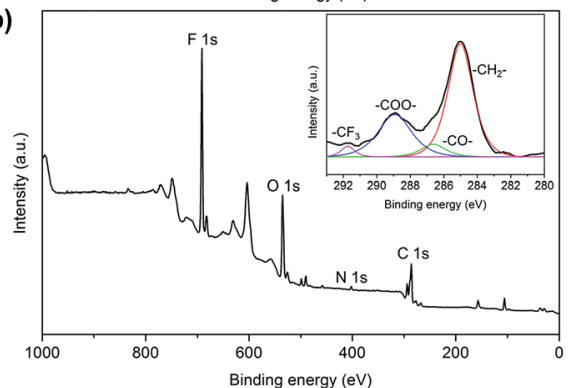

(c)

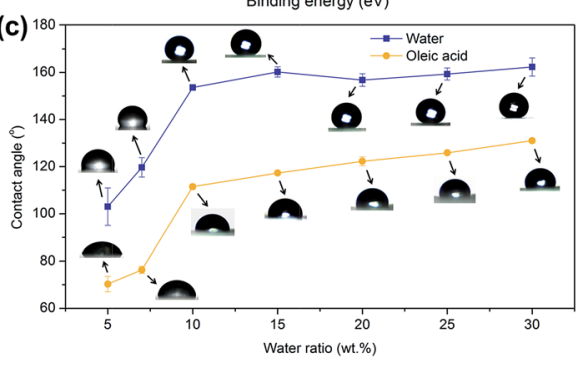

Fig. $4 \quad \mathrm{X}$-ray photoelectron spectra showing the chemical composition of the hollow cyanoacrylate nanoparticle-coated surface (a) before and (b) after silanization of the surface. (c) Water and oleic acid contact angles of superhydrophobic/oleophobic surfaces with different water ratios of the hollow cyanoacrylate nanoparticles HCNPs. Photographic images inset in (c) show the $10 \mu \mathrm{L}$ droplet of water or oleic acid on the surface.
After silanization, all samples whose WR is more than $\mathbf{1 0 . 0}$ wt\% demonstrate superhydrophobicity (Fig. 4c). This phenomenon is the result of microscopic air trapped beneath the liquid water that allows the water to roll off with only a slight tilt of the sample, which is called the Cassie state. ${ }^{40}$ However, the contact angle for oleic acid is much lower, which is owing to the lower surface tension of oleic acid than that of water. ${ }^{\mathbf{1 1}}$ Oleic acid penetrates the vacancies in the films so that air pockets beneath the oleic acid cannot be formed, which is called the Wenzel state. ${ }^{42}$ In addition, the specific surface area of the HCNP-coated surfaces becomes large and we can obtain a large value for the contact angles. As the particle diameters increase with the WR, the specific surface area of the HCNP-coated surfaces becomes large and we can obtain a large value for the contact angles. In addition, when $\mathrm{WR}=25.0$ and $30.0 \mathrm{wt} \%$, a dual micro/nano-scale roughness manifesting as a raspberrylike structure is observed (Fig. S9f-g $\dagger$ ), wherein this type of hierarchical roughness is known to be particularly favorable for repelling oil. ${ }^{\mathbf{4 3 4 4}} \mathrm{A}$ water droplet on these surfaces also exhibits high repellence, and situations showing the liquid bouncing of a superhydrophobic Cassie state are taken by a high-speed camera and are exhibited in ESI Movie S2. $\dagger$

It is confirmed from parallel transmittance values (Fig. 5b) and ultraviolet-visible spectra (Fig. 5c) that the transmittance of the films decreases as the WR increases; which is owing to the increase of the particle diameters with higher WR that thereby causes increased light scattering. The other factor decreasing the coating transparency is the aggregation of the HCNPs induced by the presence of water. However, Fig. 5b indicates that the total transmittance is considerably high, with a maximum total transmittance of $92.2 \%$ when the WR is 20.0 wt\% owing to the hollow structure of the HCNP contribution. This result suggests that this surface is transparent enough to be used in optical displays when the film is applied to a flat solid, as shown in the inset picture of Fig. 5c. Thus, this transparent superhydrophobic/oleophobic film has potential 
(a)

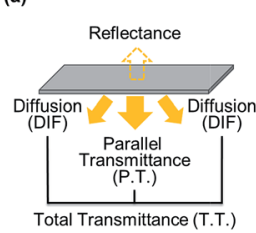

(b)

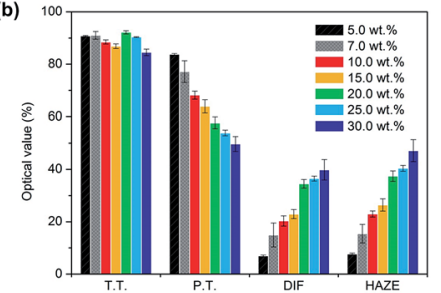

(c)

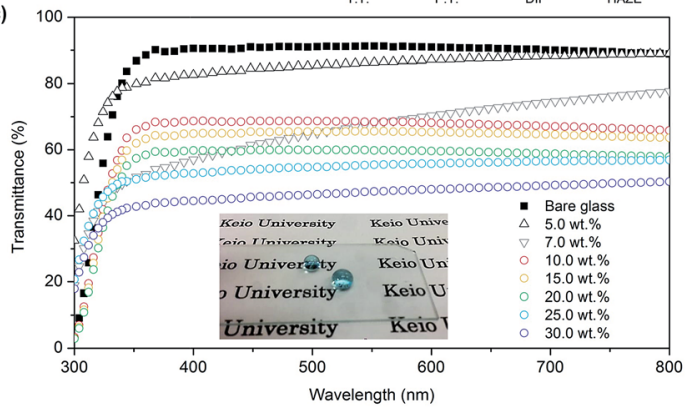

Fig. 5 (a) A schematic explaining the source of each optical value, wherein the haze, which describes the amount of light scattering when the light passes through the surfaces, is calculated by dividing diffusion by the total transmittance. (b) Total transmittance (T.T.), parallel transmittance (P.T.), diffusion (DIF), and haze (HAZE) of transparent superhydrophobic/oleophobic surfaces produced by varying water ratios. (c) The ultraviolet-visible transmission spectra of transparent superhydrophobic/oleophobic surfaces with different water ratios of the coated hollow cyanoacrylate nanoparticles. (Inset) Photograph of the transparent produced with a water ratio of $20 \mathrm{wt} \%$, exhibiting a water contact angle of $160.2^{\circ}$ and a total transmittance of $92.2 \%$.

applications for anti-fouling in solar cells and for antifingerprints in touch panel displays.

\section{Fingerprint adhesion test}

A simple anti-fingerprint test is demonstrated using a color difference meter, which measures the color coordinates followed by a calculation of the color difference values. A transparent superhydrophobic/oleophobic surface with a WR of 20.0 $\mathrm{wt} \%$ is selected as the coated glass for the test because it possesses the maximum total transmittance. Table 1 shows the color coordinates and color difference $(\Delta E)$ for bare glass and coated glass, where $L^{*}$ represents the brightness while $a^{*}$ and $b^{*}$ are the color coordinates that range from green to red and from blue to yellow, respectively. ${ }^{45}$ These color coordinates identify the location of a dot on a virtual three-dimensional sphere where the distance between two dots is the color difference, calculated as

Table 1 The color coordinates and the color differences $(\Delta E)$ of a superhydrophobic/oleophobic surface compares with bare glass before and after the adhesion of an artificial fingerprint

\begin{tabular}{|c|c|c|c|c|c|c|c|}
\hline \multirow[b]{2}{*}{ Substrate } & \multicolumn{3}{|c|}{ Initial } & \multicolumn{3}{|c|}{ After adhesion } & \multirow[b]{2}{*}{$\Delta E$} \\
\hline & $L^{*}$ & $a^{*}$ & $b^{*}$ & $L^{*}$ & $a^{*}$ & $b^{*}$ & \\
\hline Bare glass & 83.3 & -0.07 & 1.23 & 58.7 & 0.37 & 1.47 & 24.6 \\
\hline Coated glass & 83.0 & 0 & 1.40 & 72.7 & 0.13 & 1.47 & 10.2 \\
\hline
\end{tabular}

$$
\Delta E=\sqrt{\left(\Delta L^{*}\right)^{2}+\left(\Delta a^{*}\right)^{2}+\left(\Delta b^{*}\right)^{2}}
$$

A small value of $\Delta E$ means a minimal color change and a good anti-fingerprint property. The color difference in the glass measured before and after adhering an artificial fingerprint to the glass is seen to decrease in the coated glass by 14.4 compared with the bare glass, which indicates that the coated film drastically decreases fingerprint adhesion. The silanization of the surface using FAS and the texturing on the surface because of HCNP self-assembly decreases the surface tension and may contribute to the anti-fingerprint behavior of the coated surface. A lower surface tension of a solid surface means that oil, water, dust, and other materials typically present on human fingers are more likely to remain adhered to the fingers and not transferred to the solid with low surface energy. Furthermore, micro-scale pores on the roughened surface can be filled with the fingerprint oil because of its low surface tension, which may lead to the suppression of light scattering because of the fingerprint oil droplets, along with the hollow structure. These HCNP-coated oleophobic surfaces have potential practical application for touch panel displays and other optical devices (Fig. S13†).

\section{Conclusions}

Hollow cyanoacrylate nanoparticles are synthesized via the SGCIP method, which combines the instant polymerization of ECA and the nanobubbles created by a change in gas solubility. The SGCIP method is completed in a few minutes under room pressure and temperature without using any special equipment or expensive material. In addition, the diameters of the resulting HCNPs can be readily controlled by changing the ratio of water and acetone, with diameters ranging from 13 to $1830 \mathrm{~nm}$. The SGCIP method also exhibits a unique morphology transition from a network structure with $\mathrm{WR}<10 \mathrm{wt} \%$ to a nanoparticle structure with WR $>10 \mathrm{wt} \%$. The controlled HCNP structure with a low surface energy treatment demonstrates superhydrophobicity (water contact angle $>160^{\circ}$ ) and higholeophobicity (oil contact angle $>120^{\circ}$ ) with a maximum total transmittance of $92.2 \%$. The simultaneous features of oleophobicity with high total transmittance are believed to be a potential application for optical displays such as touch panels for smart phones. The facile process of synthesizing such fascinating materials may provide new insights into the design and development of functional hollow nanoparticles. In addition, our method will be useful in the development of not only transparent anti-fouling coatings but also drug delivery, heat insulator or various fields of application.

\section{Acknowledgements}

We are grateful to Dr Kouji Fujimoto whose comments and suggestions were greatly valuable throughout our study. We are indebted to Dr Yoshio Hotta, whose relevant comments were an enormous help. We also thank Prof. Sae-Hoon Kim of 
Gangneung-Wonju National University for very useful discussions. This work was partially supported by JSPS KAKENHI Grant Number 26420710.

\section{Notes and references}

1 P. B. Landon, A. H. Mo, C. Zhang, C. D. Emerson, A. D. Printz, A. F. Gomez, C. J. Delatorre, D. a M. Colburn, P. Anzenberg, M. Eliceiri, C. Oconnell and R. Lal, ACS Appl. Mater. Interfaces, 2014, 6, 9937-9941.

2 B. Peng, L. Tan, D. Chen, X. Meng and F. Tang, ACS Appl. Mater. Interfaces, 2012, 4, 96-101.

3 Z. Zhao, F. Zhu, X. Qu, Q. Wu, Q. Wang, G. Zhang and F. Liang, Polym. Chem., 2015, 6, 4144-4153.

4 G. Aguirre, J. Ramos, J. P. a. Heuts and J. Forcada, Polym. Chem., 2014, 5, 4569.

5 Y. Yang, F. Wang, Q. Yang, Y. Hu, H. Yan, Y. Chen, H. Liu, G. Zhang, J. Lu, H. Jiang and H. Xu, ACS Appl. Mater. Interfaces, 2014, 6, 18163-18171.

6 C. Li, A. C. Jamison, S. Rittikulsittichai, T. Lee and T. R. Lee, ACS Appl. Mater. Interfaces, 2014, 6, 19943-19950.

7 M. Fujiwara, K. Shiokawa, H. Monobe and Y. Shimizu, ACS Appl. Mater. Interfaces, 2015, 7, 1107-1113.

8 T. Liu, W. Zhang, T. Song, X. Yang and C. Li, Polym. Chem., 2015, 6, 3305-3314.

9 F. Wang, L. Huang, J. Li, L. Lin, Z. Liu and Z. Dong, Polym. Chem., 2014, 5, 4332.

10 F. Caruso, R. A. Caruso, H. Mo and H. Mohwald, Science, 1998, 282, 1111-1114.

11 Y. Du, L. E. Luna, W. S. Tan, M. F. Rubner and R. E. Cohen, ACS Nano, 2010, 4, 4308-4316.

12 S. Bakalyar, M. Bradley and R. Honganen, J. Chromatogr., 1978, 158, 277-293.

13 M. Tenjimbayashi and S. Shiratori, J. Appl. Phys., 2014, 116, 114310.

14 A. Yaghmaei, M. K. Torbati and S. M. Zebarjad, J. Vinyl Addit. Technol., 2010, 16, 204-208.

15 X. Tang, T. Wang, F. Yu, X. Zhang, Q. Zhu, L. Pang, G. Zhang and M. Pei, RSC Adv., 2013, 3, 25670-25673.

16 L. Xu, J. He and L. Yao, J. Mater. Chem. A, 2014, 2, 402-409. 17 Q. Fu, X. Wu, D. Kumar, J. W. C. Ho, P. D. Kanhere, N. Srikanth, E. Liu, P. Wilson and Z. Chen, ACS Appl. Mater. Interfaces, 2014, 6, 20685-20692.

18 N. Wang, D. Xiong, Y. Deng, Y. Shi and K. Wang, ACS Appl. Mater. Interfaces, 2015, 7, 6260-6272.

19 K. Zhou, Q. G. Zhang, H. M. Li, N. N. Guo, A. M. Zhu and Q. L. Liu, Nanoscale, 2014, 6, 10363-10369.

20 K. Rohrbach, Y. Li, H. Zhu, Z. Liu, J. Dai, J. Andreasen and L. Hu, Chem. Commun., 2014, 50, 13296-13299.
21 X. Gao, L.-P. Xu, Z. Xue, L. Feng, J. Peng, Y. Wen, S. Wang and X. Zhang, Adv. Mater., 2014, 26, 1771-1775.

22 S. Lee, D. Ham, D. Lee, H. Bong and K. Cho, Langmuir, 2013, 29, 15051-15057.

23 X. Tang, S. Nan, T. Wang, Y. Chen, F. Yu, G. Zhang and M. Pei, RSC Adv., 2013, 3, 15571-1557524.

24 K. Fukada, S. Nishizawa and S. Shiratori, J. Appl. Phys., 2014, 115, 103516.

25 J. Zhang and S. Seeger, Angew. Chem., Int. Ed. Engl., 2011, 50, 6652-6656.

26 Y. Huang, Y. Jen, L. Chen, K. Chen and S. Chattopadhyay, ACS Nano, 2015, 9, 301-311.

27 A. Yoshida, M. Motoyama, A. Kosaku and K. Miyamoto, Zool. Sci., 1997, 14, 737-741.

28 L. Xu and J. He, Langmuir, 2012, 28, 7512-7518.

29 L. Gao and J. He, J. Colloid Interface Sci., 2013, 396, 152-159.

30 K. Manabe, S. Nishizawa, K. Kyung and S. Shiratori, ACS Appl. Mater. Interfaces, 2014, 6, 13985-13993.

31 T. Makuta, Y. Yoshihiro, T. Sutoh and K. Ogawa, Mater. Lett., 2014, 131, 310-312.

32 J. Kreuter, R. N. Alyautdin, D. A. Kharkevich and A. A. Ivanov, Brain Res., 1995, 674, 171-174.

33 S. Dahl, Å. Fredenslund and P. Rasmussen, Ind. Eng. Chem. Res., 1991, 30, 1936-1945.

34 X. Du, J. S. Li, L. X. Li and P. a. Levkin, J. Mater. Chem. A, 2013, 1, 1026-1029.

35 H. G. M. Edwards and J. S. Day, J. Raman Spectrosc., 2004, 35, 555-560.

36 S. K. Tomlinson, O. R. Ghita, R. M. Hooper and K. E. Evans, Dent. Mater., 2007, 23, 799-806.

37 P. G. Bowers, K. Bar-Eli and R. M. Noyes, J. Chem. Soc., Faraday Trans., 1996, 92, 2843-2849.

38 A. Perera and F. Sokolić, J. Chem. Phys., 2004, 121, 1127211282.

39 N. Al Khouri Fallouh, L. Roblot-Treupel, H. Fessi, J. P. Devissaguet, F. Puisieux and N. Al Khouri, Int. J. Pharm., 1986, 28, 125-132.

40 A. Cassie and S. Baxter, Trans. Faraday Soc., 1944, 40, 546551.

41 I. Okada and S. Shiratori, ACS Appl. Mater. Interfaces, 2014, 6, 1502-1508.

42 R. N. Wenzel, Ind. Eng. Chem., 1936, 28, 988-994.

43 S. Nishizawa and S. Shiratori, Appl. Surf. Sci., 2012, 263, 8-13. 44 Z. Geng and J. He, J. Mater. Chem. A, 2014, 2, 16601-16607. 45 W. Navarrini, T. Brivio, D. Capobianco, M. V. Diamanti, M. Pedeferri, L. Magagnin and G. Resnati, J. Coat. Technol. Res., 2011, 8, 153-160. 